\title{
Thioacetamide induced liver damage in zebrafish embryo as a disease model for steatohepatitis
}

\author{
Aseervatham Anusha Amali ${ }^{1}$, Ravikumar Deepa Rekha ${ }^{1}$, Cliff Ji-Fan Lin ${ }^{1,2}$, \\ Wei-Lun Wang ${ }^{1,3}$, Hong-Yi Gong ${ }^{1}$, Gour-Mour Her ${ }^{4} \&$ Jen-Leih Wu ${ }^{1, *}$ \\ ${ }^{1}$ Laboratory of Marine Molecular Biology and Biotechnology 301, Institute Cellular and Organismic Biology, \\ Academia Sinica, 128, Academia Road, Section 2, NanKang, Taipei, 11529, Taiwan; ${ }^{2}$ Graduate Institute of \\ Life Sciences, National Defense Medical Center, Taipei, Taiwan; ${ }^{3}$ Institute of Fisheries Sciences, National \\ Taiwan University, Taipei, Taiwan; ${ }^{4}$ Graduate Institute of Biotechnology, National Taiwan Ocean University, \\ Keelung, Taiwan
}

(C) 2006 National Science Council, Taipei

Key words: liver, steatosis, thioacetamide, zebrafish

\section{Summary}

Steatohepatitis has recently been increasing as a cofactor influencing the progression of fibrosis, cirrhosis, adenoma and carcinoma in liver; however, the mechanisms by which it contributes to liver injury remain uncertain. We induced steatohepatitis in zebrafish embryos using thioacetamide (TAA). TUNEL assay revealed significant increasing of apoptosis in liver after 5 days post fertilization and the increasing of apoptosis was observed to be associated with the up-regulation of apoptotic genes such as, bad, bax, P-38a, caspase-3 and 8, and JNK-1. Histological sections by oil red O stain showed the accumulation of fatty droplets which causes the pushing of the nucleus towards one side. Up-regulation of steatosis markers such as, ACC, adiponectin, PTL, CEBP- $\alpha$ and $\beta$, SREBP-1 was also observed. Furthermore, the elevation of glutathione peroxidase in TAA treated embryos indicated that TAA induces lipid peroxidation which leads to causes liver damage. Zebrafish has already been considered as a good human disease model and in this context; TAA-treated zebrafish may serve as a good animal model to study the molecular pathogenesis of steatohepatitis. Moreover, non-availability of specific drugs to prevent steatohepatitis, this animal model may serve as a powerful preclinical platform to study the therapeutic strategies and for evaluating chemoprevention strategies for this disease.

\section{Introduction}

Steatohepatitis is an increasingly recognized condition that may progress to the end stage of liver disease. Fatty liver disease is a clinicopathological disease which ranges from simple triglyceride accumulation in hepatocytes (hepatic steatosis) to hepatic steatosis with inflammation (steatohepatitis), fibrosis, and cirrhosis. Steatohepatitis represents only a stage within the spectrum of

*To whom correspondence should be addressed. Tel: +886-227899568; Fax: +886-2-27824595, E-mail: jlwu@gate.sinica. edu.tw nonalcoholic fatty liver disease. The hepatic steatosis is due to the accumulation of lipids, predominantly triglycerides, within the hepatocytes due to variable combinations of excess lipid synthesis and uptake with altered intermediate metabolism and secretion. The factors important in the development of steatohepatitis are fatty acids, cytokines (e.g., adiponectin, leptin, and resistin from adipose tissue and TNF-alpha and TGF-beta from kuppfer cells), and the metabolic activation of enzyme pathways in the hepatocytes.

Hepatic steatosis is a prerequisite for subsequent events that lead to liver injury. There are no 
animal models to incorporate all features of human steatohepatitis and the availability of noninvasive techniques to study hepatic metabolism in humans are limited. Moreover, liver biopsies are required to identify individuals with nonalcoholic steatohepatitis (NASH), which precludes large population based studies. Steatosis occurs because the liver metabolizes free fatty acids, which are partly mediated by lipase. Lipase is inhibited by insulin, and persons with insulin resistance have elevated serum insulin levels. Free fatty acids accumulated in the liver are oxidized by mitochondria and used for the formation of triglycerides and fat accumulation in the liver [1].
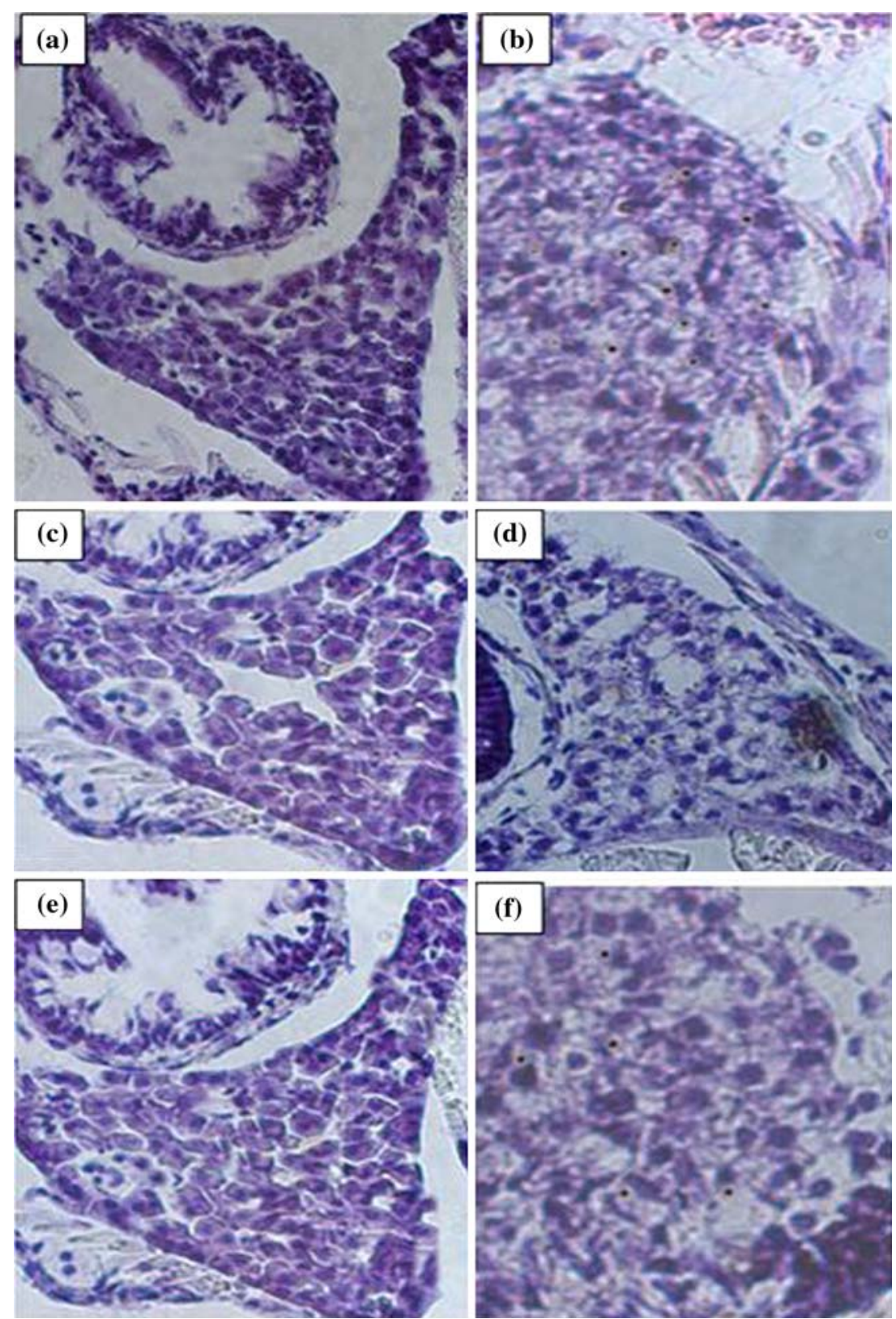

Figure 1. In situ Detection of DNA fragmentation by TUNEL assay. The DNA fragmentation (brown nucleus) indicates TUNEL positive cells. (a) $3 \mathrm{dpf}$ control liver, (b) $3 \mathrm{dpf}$ embryo treated with TAA, (c) $5 \mathrm{dpf}$ control liver, (d) 5 dpf embryo treated with TAA, (e) $7 \mathrm{dpf}$ control liver, (f) $7 \mathrm{dpf}$ embryo treated with TAA. 

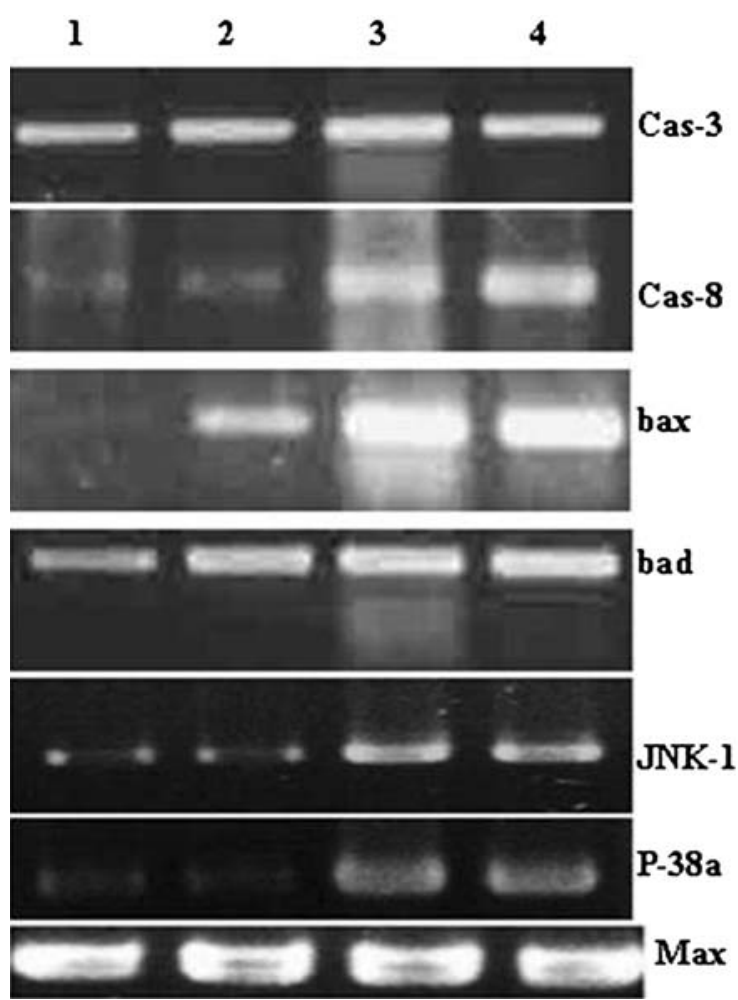

Figure 2. Expression profile of different apoptotic related genes in control and 10 days TAA treated embryos. (1) control embryos, (2) 3-days-old treated embryos, (3) 5 days treated embryos, (4) 7 days treated embryos. Max was used as an internal control.

Steatosis can be induced by thioacetamide (TAA). The results of TAA induced steatosis with other heptotoxicant models have been highly heterogenous. According to the pattern and doses of administration, TAA induces acute liver failure [2] or hepatic cirrhosis [3]. Liver cirrhosis induced by TAA is associated with the exacerbation of lipid peroxidation and the depletion of antioxidant status [4].

TAA was originally used to preserve oranges is a potent hepatotoxin and carcinogen $[5,6]$. Prolonged administration of TAA causes hyperplastic liver nodules, liver cell adenomas and hepatocarcinomas. Various TAA induction methods have been tried in experimental animals to produce liver cirrhosis and tumors, including intraperitoneal or subcutaneous administration $[7,8]$ and administering toxin with food [3] or drinking water [9]. TAA is known as a potent hepatotoxicant which requires metabolic activation by missed function oxidases. Cytochrome
P450 2B, 2E1 and flavin monooxygenase metabolize TAA to its toxic metabolites [10]. TAA is also known to cause toxic effects in the pancreas [11] and kidney [6]. Although the molecular mechanisms that cause these toxic effects in different organs are not understood, TAA is known to interfere with ribosomal activity, thus hindering protein synthesis [11]. TAA administration has also been shown to stimulate DNA synthesis [12]. The current investigation presents an understanding of mechanism of the steatohepatitis disease using zebrafish as a simple and effective animal model. Moreover embryos with green liver [13] were used through out our study.

\section{Materials and methods}

\section{Animals and chemical treatment}

Zebrafish used in these studies were, established as a transgenic line in our lab using liver fatty acid binding protein (LFABP) promoter [13, 14]. The fish were maintained at 25 to $28{ }^{\circ} \mathrm{C}$, exposed to 12 $\mathrm{h}$ light and dark periods, and fed twice a day.

Fertilized eggs were maintained in the freshwater tank at $28{ }^{\circ} \mathrm{C}$, and the developing embryos were collected and maintained at $28^{\circ} \mathrm{C}$. Developmental stages were classified [15], and the embryos were observed under the microscope. After $72 \mathrm{~h}$ the embryos were divided into five groups and each group were treated with $0.025 \%$ TAA. The mortality rate was observed daily.

\section{Apoptosis assay}

To check the cell death in the liver of zebrafish larvae, we used the TUNEL Assay. The embryos were fixed in $4 \%$ paraformaldehyde and tissue sections $(10 \mu \mathrm{m})$ were prepared, and terminal deoxynucleotidyl transferase dUTP nick-end labeling (TUNEL) assay was performed following manufacturer's instructions (in situ cell death detection kit; Roche Molecular Biochemicals, Mannheim, Germany). Apoptosis bodies were detected using a DAB substrate.

\section{Optics}

For analysis of the GFP fluorescence patterns, embryos and larvae were either anesthetized with 

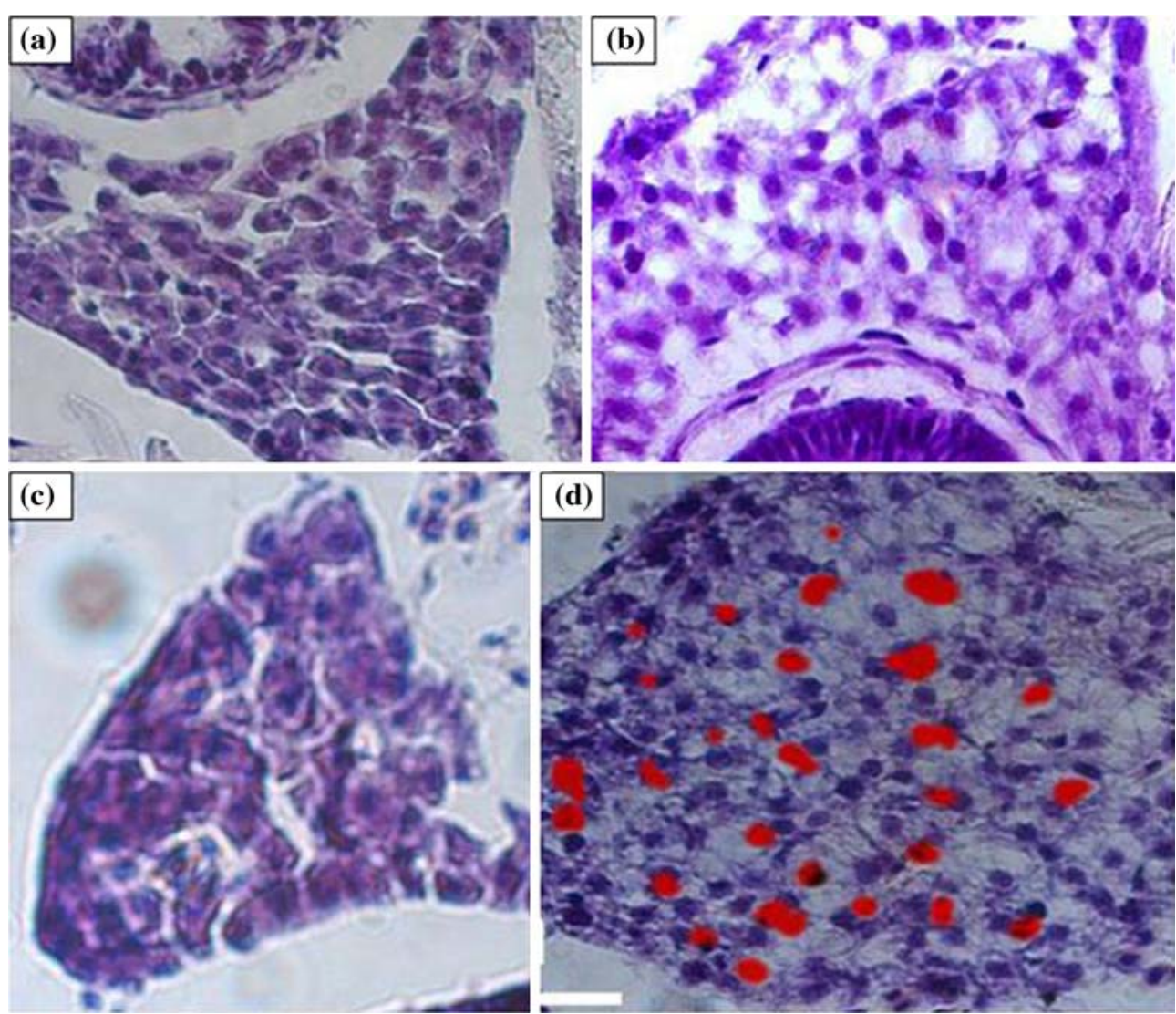

Figure 3. Liver sections of embryos treated with TAA for 10 days. (a) control $10 \mathrm{dpf}$ embryo (H\&E staining), (b) $10 \mathrm{dpf}$ embryos treated with TAA (H\&E), (c) control 10 dpf embryo, (d) 0 dpf embryos treated with TAA. Lipid accumulation was confirmed by oil red $\mathrm{O}$ staining.

$168 \mathrm{mg} / \mathrm{ml} \mathrm{3-aminobenzoic} \mathrm{acid} \mathrm{ethyl} \mathrm{ester} \mathrm{(Sig-}$ ma) or fixed overnight at $4{ }^{\circ} \mathrm{C}$ in PBS containing $4 \%$ paraformaldehyde and then washed with PBS. For analyzing GFP fluorescent patterns, embryos and larvae were anesthetized with $0.05 \%$ 2-phenoxyethanol (Sigma) and GFP expression was examined using a filter $(480 \mathrm{~nm}$ excitation, $505 \mathrm{~nm}$ emission) set on an ECLIPSE E600 microscope (Nikon) equipped with the DXM 1200 CCD camera (Nikon). For fluorescence imaging by confocal laser scanning microscopy (CLSM), we used a Leica TCSNT system fitted to a Leica microscope with a $20 \times$ objective (Nikon).

\section{$R T-P C R$}

Total RNA was isolated from different tissues by using TRIZOL (Boehringer Mannheim), accord- ing to the manufacturer's instruction. First-strand cDNA was synthesized in a $20 \mu \mathrm{l}$ RT reaction from $5 \mu \mathrm{g}$ of total RNA by using Thermoscript RT-PCR system (Invitrogen). PCR was performed with $2 \mu \mathrm{l}$ of the RT reaction. The PCR program was $94{ }^{\circ} \mathrm{C}, 30 \mathrm{~s}, 55^{\circ} \mathrm{C}$ for $30 \mathrm{~s}$, and $72{ }^{\circ} \mathrm{C}$ for $1 \mathrm{~min}$ and performed 35 cycles. The final extension time was $7 \mathrm{~min}$ at $72{ }^{\circ} \mathrm{C}$. The PCR products were subjected to $3 \%$ gel electrophoresis.

\section{Histopathological examination}

Liver from wild type and wild type treated with TAA zebrafish embryos was fixed in $4 \%$ paraformaldehyde and embedded in agarose, and cryosectioned $(10 \mu \mathrm{m})$. The sections were routinely stained with haematoxylin and eosin. To find out the fatty droplet accumulation the sections were stained with oil red $\mathrm{O}$. 


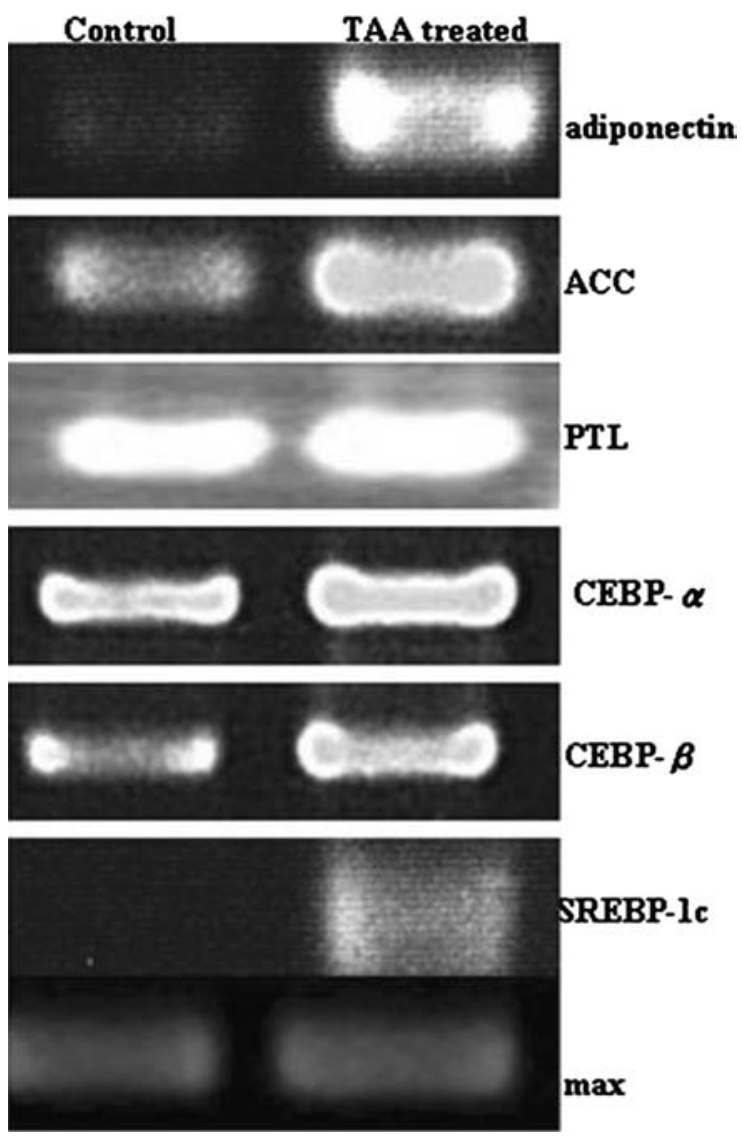

Figure 4. RT-PCR for the expression pattern of different lipogenic, adipoenic related genes in control and TAA treated embryos.

\section{Results}

\section{TAA induce apoptosis in zebrafish embryos}

The liver damage in zebrafish model was induced by adding TAA in the rearing water of the embryos. The exposure of the embryos to TAA for a few hours produces apoptosis. The apoptotic hepatocytes stained with the TUNEL method were observed in the TAA-induced liver sections (Figure 1a-f). Zebrafish embryos after continuous exposure for 3,5 and 7 days were collected and examined for the presence of apoptotic bodies. We have observed the apoptotic bodies after 3 days of exposure (Figure 1b) and the number of these bodies increased significantly on $5 \mathrm{~d}$ (Figure 1d), however, the number of these apoptotic bodies started to diminish on 7 days (Figure 1f), which may be because of more number of necrotic bodies being replaced and showing the progression of the disease. No stained hepatocytes were observed in control (Figure 1a,c,e). The apoptosis was confirmed by RT-PCR using major apoptotic markers namely, caspase 3, caspase 8, JNK 1, Bad, Bax, and P38a (Figure 2). These results were also correlated with that of the TUNEL assay, showing the occurrence of apoptosis during early days of exposure.

\section{Histopathological observation and Steatohepatitis in TAA treated embryos}

A histological study was carried out on the hepatocytes of 10 days old TAA-treated and control embryos. In control, the hepatic cells showed well preserved cytoplasm and prominent nucleus (Figure 3a). Liver sections of TAA-treated animals showed hepatic cells with necrosis along with various grade of fatty changes comprising of tiny and large vacuoles (Figure $3 \mathrm{~b}$ ). The oil red $\mathrm{O}$ staining (Figure 3c,d) revealed that the vacuoles contain lipids. The lipid droplet accumulation in the hepatocytes pushed the nucleus to one side of the cell.

\section{Expression of adipogenic and lipogenic genes in TAA-treated embryos}

To investigate the triglyceride accumulation in TAA treated livers, we measured the expression of adipognic, lipogenic and $\beta$-oxidation related genes (Figure 4). RT-PCR analysis revealed dramatic induction of adipocyte specific gene adiponectin. Expression levels of CEBP- $\alpha$ and $\beta$ were upregulated very mildly. Moderate increase was observed for $\beta$-oxidation related gene PTL. Elevation of SREBP1-c, another transcription factor that regulates lipid synthesis was observed in TAA treated embryos. Fatty acid modifying enzyme ACC downstream of SREBP was also increased in the treated embryos.

\section{Reduction of GFP intensity in TAA treated embryos}

The 5'-flanking sequences of the zebrafish L-FABP gene and used a green fluorescent protein was isolated and (GFP) transgenic strategy to generate liver-specific transgenic zebrafish [13]. The cryosection of these transgenic green liver embryos treated with TAA showed many vacuoles and the 
(a)

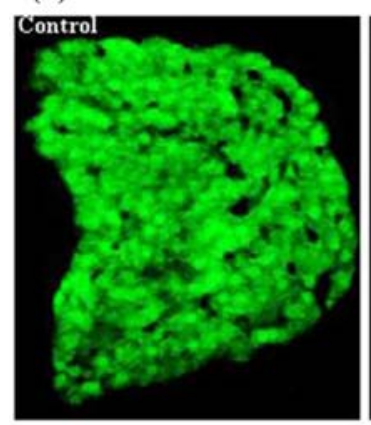

(b)

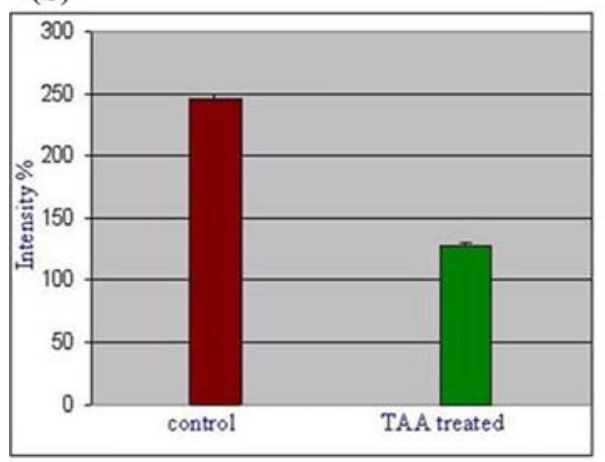

TAA treated

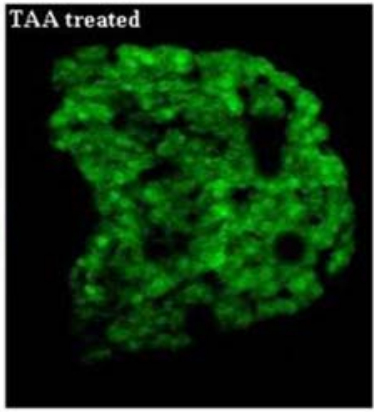

(c)

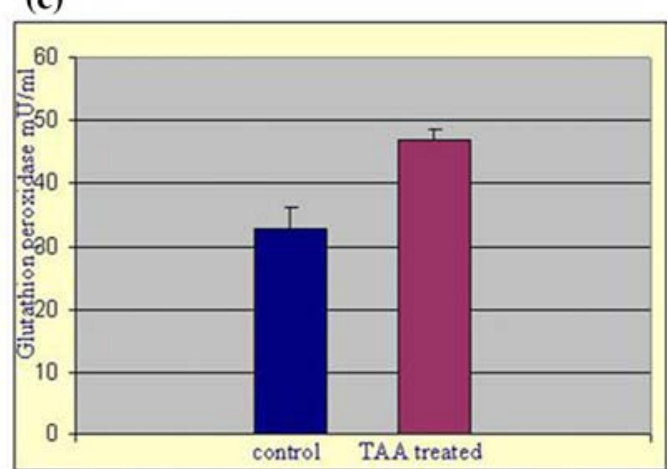

Figure 5. (a) Section showed the morphology of hepatocyte. Vacuoles were observed in TAA treated embryos. (b) Graph showing the intensity GFP in control and embryos treated with TAA. (c) Diagram showed increased GSH peroxidation.

intensity of GFP also reduced than the control (Figure 5b). In control, the hepatocytes are packed densely (Figure 5a).

\section{Decrease in glutathione peroxidase in TAA treated embryos}

Increased production of ROS by hepatocytes has been associated with the HCC development. Glutathione peroxidase level increased in TAA treated embryos than the control (Figure 5c). The decrease in the antioxidant shows that the embryo has lost its defense and hence the disease starts its progression slowly.

\section{Discussion}

In the present study, chronic administration of TAA caused liver steatosis as indicated by histological changes, molecular and biochemical analysis. TAA was chosen as a reference hepatotoxin for this study as it is known to induce both apoptosis and necrosis, depending on the dose and time of treatment [2]. We showed that treatment in embryos with $0.025 \%$ TAA for 3 days resulted in the formation of characteristic mild apoptotic hepatocytes and it increases on 5 days in the centrilobular zones. The characteristic morphological aspect of apoptotic cells was observed in the liver sections: cell shrinkage of single scattered cells, margination and clumping of nuclear chromatin, detachment of neighboring cells and development on the cell surface of blebs which lead to the formation of apoptotic bodies. Moreover, the molecular markers for apoptosis like caspase-3, caspase- 8 bad and bax, JNK-1, P38a were upregulated. TAA is known to be metabolized to a reactive metabolite TAA S-oxide by CYP2E1 localized in the centrilobular zones [16]. TAA S oxide then binds covalently to macromolecules in the centrilobular zones and induce cellular damages $[17,16,18]$, which in turn trigger apoptosis. The fact that apoptosis was specifically retrieved in the centrilobular zone of rat liver slices treated with TAA is in agreement with previously published data showing that the maintenance and localization of CYP isoforms is maintained in this 
model $[19,20]$. Interestingly, apoptotic hepatocytes were localized in both centrilobular and periportal areas when the embryos were treated with TAA. This could be explained by the fact that the lesions were more severe with diffuse disorganization of the parenchyma, possible necrosis and diffusion of reactive metabolites, and subsequent loss of regional specificity for the formation of apoptotic hepatocytes.

In this study, we found that increasing steatosis in TAA treated embryos. Accumulations of lipid in nonadipose tissues have been reported to lead to cell death. Several reports showed that chronic exposure of TAA induces steatosis, fibrosis, cirrhosis and leads to hepatocellular carcinoma. In rats [21] cytochrome p-450 system is known to metabolize thioactamide in the liver. Formation of thioacetamide-5-oxide which is responsible for the changes in cell permeability, increase intracellular concentration of calcium, increase in nuclear volume and enlargement of nucleoli and also inhibit mitochondrial activity which leads to cell death $[22,23,24]$. As a result, the embryos exhibit steatohepatitis similar to that of observed in humans with nonalcoholic steaohepatitis. A net retention of lipids within the hepatocytes, mostly in the form of triglyceride, is a prerequisite for the development of fatty liver disease [25]. The primary metabolic abnormalities leading to lipid accumulation are not well understood. Our results revealed that three was an upregulation of genes involed in lipid metabolism. Acetyl-CoA carboxylase (ACC) was upregulated in the treated embryos. It converts acetyl-Coa to malonyl CoA, which is used by fattyacid synthase to form Palmitic acid. Activation of lipogenesis is transcriptionally activatd by membrane bound transcription factors Sterol regulatory element-binding protein-1 (SREBP-1) [26, 27]. In the nucleus, SREBP-1 activate all the genes involved in lipogenesis [28].

Oxidative stress results from an imbalance between pro-oxidant and anti-oxidant chemical species that leads to oxidative damage of cellular macromolecules [29]. TAA treatment induces fatty acid accumulation and also TAA influence cytochrome-450 (CYP) enzymes, which form ROS. ROS induces oxidative stress and cell death via ATP and NADP depletion, DNA and protein damage, and glutathione depletion. Finally ROS and products of lipid peroxidation can lead to fibrosis. Glutathione is an important endogenous antioxidant system that is found in particularly high concentration in liver and is known to have a key function protective process. The reduced forms of GSH become readily oxidized to GSSG on interacting with free radicals. Excessive production of free radicals resulted oxidative stress, which leads to damage of macromolecules like lipids, and can also induce lipid peroxidation in vivo [30].The peroxisomal $\beta$-oxidation system consist of the $\mathrm{H}_{2} \mathrm{O}_{2}$ generating enzymes AOX, and PTL [31]. Disappropriate increases in these $\mathrm{H}_{2} \mathrm{O}_{2}$ generating enzymes coupled with reductions in the $\mathrm{H}_{2} \mathrm{O}_{2}$ generating enzymes catalase and glutathione peroxidase, may lead to sustained oxidative stress in liver [32]. As a result, hepatocytes may progressively accumulate lipofusion, by product of ROS induced lipid peroxidation.

Steatohepatitis affects a large proportion of the world's population. Hepatotoxins, insulin resistance and oxidative stress have critical roles in the pathogenesis of fatty liver disease. No effective medical therapy is currently available for patients with steatoepatitis. So after treating the embryo with TAA it provides a good model to study the mechanism of formation steatohpatitis and it is a good platform for the drug screening that leads to drug discovery.

\section{References}

1. Reid A.E., Nonalcoholic steatohepatitis. Gastroenterology. 121: 710-723, 2001.

2. Ledda-Columbano G.M., Coni P., Curto M., Giacomini L., Faa G., Oliverio S., Piacentini M. and Columbano A., Induction of two different modes of cell death, apoptosis and necrosis, in rat liver after a single dose of thioacetamide. Am. J. Pathol. 139: 1099-1109, 1991.

3. Muller D., Zimmerman S.I. and Schiller F., Drug metabolism in rat liver injured by thioacetamide. Arch. Toxicol. 5: 368-371, 1982.

4. Cruz A., Padillo F.J., Torres E., Navarrete C.M., MunozCastaneda J.R., Caballero F.J., Briceno J., Marchal T., Tunez I., Montilla P., Pera C. and Muntane J., Melatonin prevents experimental liver cirrhosis induced by thioacetamide in rats. J. Pineal Res. 39: 143-150, 2005.

5. Kuriyama S., Tsujimoto T., Nakatani Y., Tsujinoue H., Yoshiji H., Mitoro A., Yamazaki M., Okuda H., Toyokawa Y., Nagao S., Nishiwaki I. and Fukui H., Sonographic estimation of liver tumor development induced by oral administration of thioacetamide in rat. In Vivo. 3: 129-134, 1999.

6. Al-Bader A.A., Mathew T.C., Al-Mosawi M., Dashti H.M., Kumar D. and Singal P.K., Thioacetamide induced 
changes in trace elements and kidney damage. J. Trace Elem. Exp. Med. 12: 1-14, 2003.

7. Dashti H., Jeppsson B., Hagerstrand I., Hultberg B., Srinivas U., Abdulla M., Joelsson B. and Bengmark S., Early biochemical and histological changes in rats exposed to a single injection of thioacetamide. Pharmacol. Toxicol. 60: 171-174, 1987.

8. Gallagher C.H., Gupta D.N., Judah J.D. and Rees K.R., Biochemical changes in liver in acute thioacetamide intoxication. J. Pathol. Bacteriol. 72: 193-201, 1956.

9. Dashti H.M., Mathew T.C., Jadaon M.M. and Ashkanani E., Zinc and liver cirrhosis: biochemical and histopathologic assessment. Nutrition 13: 206-212, 1997.

10. Lee J.W., Shin K.D., Lee M., Kim E.J., Han S.S., Han M.Y., Ha H., Jeong T.C. and Koh W.S., Role of metabolism by flavin-containing monooxygenase in thioacetamide-induced immunosuppression. Toxicol. Lett. 136: 163-172, 2003.

11. Barker E.A. and Smuckler E.A., Altered microsome function during acute thioacetamide poisoning. Mol. Pharmacol. 8: 318-326, 1972.

12. Morley C.G. and Boyer J.L., Stimulation of hepatocellular proliferation by a serum factor from thioacetamide-treated rats. Biochim. Biophys. Acta. 477: 165-176, 1977.

13. Her G.M., Chiang C.C., Chen W.Y. and Wu J.L., In vivo studies of liver-type fatty acid binding protein (L-FABP) gene expression in liver of transgenic zebrafish (Danio rerio). FEBS Lett. 538: 125-133, 2003.

14. Her G.M., Yeh Y.H. and Wu J.L., 435-bp liver regulatory sequence in the liver fatty acid binding protein (L-FABP) gene is sufficient to modulate liver regional expression in transgenic zebrafish. Dev. Dyn. 227: 347-356, 2003.

15. Westerfield M., The Zebrafish Book: A Guide for the Laboratory Use of Zebrafish (Danio rerio), 2nd ed., University of Oregon Press, Eugene, 1994.

16. Ramaiah S.K., Apte U. and Mehendale H.M., Cytochrome P4502E1 induction increases thioacetamide liver injury in dietrestricted rats. Drug Metab. Dispos. 29: 1088-1095, 2001.

17. Hunter A.L., Holscher M.A. and Neal R.A., Thioactamide induced hepatic necrosis. Involvement of the mixed function oxidase enzyme system. J. Pharmacol. Exp. Ther. 200: 439-448, 1977.

18. Witzma F.A., Fultz C.D., Mangipudy R.S. and Mehendale H.M., Two-dimensional electrophoretic analysis of compartment-specific hepatic protein charge modification induced by thioacetamide exposure in rats. Fundam. Appl. Toxicol. 31: 124-132, 1996.

19. Martin H., Sarsat J.P., deWaziers I., Housset C., Balladur P., Beaune P., Albaladejo V. and Lerche-Langrand C., Induction of cytochrome P450 2B6 and 3A4 expression by
Phenobarbital and cyclophosphamide in cultured human liver slices. Pharm. Res. 20: 557-568, 2003.

20. Meredith C., Scott M.P., Renwick A.B., Price R.J. and Lake B.G., Studies on the induction of rat hepatic CYP1A, CYP2B, CYP3A and CYP4A subfamily form mRNAs in vivo and in vitro using precision-cut rat liver slices. Xenobiotica 33: 511-527, 2003.

21. Chieli E. and Malvadi G., Role of Cyt P-450 dependent and FAA containing mono oxygenases in the bioactivation of thioacetamide, thiobezamide and their sulophoxides. Biochem. Pharmacol. 34: 395-396, 1985.

22. Ambrose A.M., DeEds F. and Rather L.J., Toxicity of thioacetamide in rats. J. Indian Hygiene Toxicol. 31: 158161, 1949.

23. Neal R.A. and Halpert J., Toxicology of thionosulfer compounds. Annu. Rev. Pharmacol. Toxicol. 22: 321-339, 1982.

24. Unger R.H. and Orci L., Lipoapoptosis: its mechanism and its diseases. Biochim. Biophy. Acta. 1585: 202-212, 2002.

25. Marchesini G., Brizi M., Morselli-Labate A.M. et al., Association of non lcoholic fatty liver disease with insulin resistance. Am. J. Med. 107: 450-455, 1999.

26. Shimomura I., Bashmakov Y., Ikemoto S., Horton J.D., Brown M.S. and Goldstein J.L., Insulin selectively increases SREBP-1c mRNA in the livers of rats with treptozotocin-induced diabetes. Proc. Natl. Acad. Sci. USA. 96: 13656-13661, 1999.

27. Foretz M., Guichard C., Ferre P. and Foufelle F., Sterol regulatory element binding protein-1c is a major mediator of insulin action on the hepatic expression of glucokinase and lipogenesis-related genes. Proc. Natl. Acad. Sci. USA. 96: 12737-12742, 1999.

28. Horton J.D., Goldstein J.L. and Brown M.S., SREBPs: activators of the complete program of cholesterol and fatty acid synthesis in the liver. J. Clin. Invest. 109: 1125-1131, 2002.

29. Robertson G., Leclercq I. and Farrell G.C., Nonalcoholic steatosis and steatohepatitis: II. Cytochrome P-450 enzymes and oxidative stress. Am. J. Physiol. Gastrointest. Liver Physiol. 281: G1135-G1139, 2001.

30. Davila J.C., Lenherr A. and Acosta D., Protective effects of flavonoids on drug induced hepatotoxicity. Toxicology 57: 267-268, 1989.

31. Reddy J.K. and Hashimoto T., Peroxisomal beta-oxidation and peroxisome proliferator-activated receptor alpha: an adaptive metabolic system. Annu. Rev. Nutr. 21: 193-230, 2001

32. Gonzalez F.J., Peters J.M. and Carttley R.C., Mechanism of action of the nongenotoxic peroxysome proliferators activator receptor alpha. J. Natl. cancer Inst. 90: 17021709, 1998. 